\title{
Amélioration de la qualité des sols acides de Lubumbashi (Katanga, RD Congo) par l'application de différents niveaux de compost de fumiers de poules
}

\author{
Yannick Useni Sikuzani1 ${ }^{\star}$, Glady Mwamba llunga1, Theodore Mwamba Mulembo¹, Becker Ntumba \\ Katombe2, Jonas Lwalaba Wa Lwalaba1, Mick Assani Bin Lukangila1', Antoine Kanyenga Lubobo1, \\ Louis Baboy Longanza ${ }^{1}$ \\ ${ }^{1}$ Département de Phytotechnie, Faculté des Sciences agronomiques, Université de Lubumbashi, Lubumbashi, RD \\ Congo. BP 1825 ; \\ 2Section Gestion de l'eau et fertilité du sol, Antenne Gestion des Ressources Naturelles, Institut National pour \\ l'Etude et la Recherche Agronomiques, Station de Kipopo, RD Congo BP 224 \\ *Auteur correspondant : yannickuseni@gmail.com
}

Original submitted in on 6th May 2014. Published online at www.m.elewa.org on 31st May 2014. http://dx.doi.org/10.4314/jab.v77i1.3

\section{RESUME}

Objectifs : Une expérimentation a été conduite sur un sol acide pour évaluer l'influence des composts et des engrais minéraux sur les propriétés chimiques de ce sol.

Méthodologie et résultats : L'essai a été installé suivant un dispositif complètement randomisé avec six répétitions de six traitements: T0 (témoin non fertilisé); T1 (175 kg NPK+87 kg Urée par hectare); T2 (double de T1, soit $350 \mathrm{~kg} \mathrm{NPK}+175 \mathrm{~kg}$ urée); T3 (15 tha-1 de compost), T4 (double de T3, soit $30 \mathrm{t}^{-\mathrm{ha}^{-1}} \mathrm{de}^{-1}$ compost); T5 (quadruple de T3, 60 t.ha $^{-1}$ de compost). Avant l'installation de l'essai, un compostage de fumier de poules a été effectué pendant 36 jours. A la fin de l'expérimentation, des analyses chimiques ont été réalisées sur des échantillons de sol et de compost de fumiers de poules. Les teneurs en cations échangeables semblent plus élevées par rapport aux valeurs de référence trouvées par d'autres auteurs dans la zone d'étude. Les valeurs élevées trouvées pour ces paramètres dans le site de la présente étude pourraient provenir de l'apport des fertilisants. Par contre, sur le témoin, l'on a noté une faible disponibilité des éléments nutritifs. En outre, les niveaux moyens de $K, P, C$ et $p H$ sont élevés dans les pots amendés avec de grandes quantités de composts. Les différences significatives ont été observées entre les différentes doses des composts utilisés et des engrais minéraux NPK sur les caractéristiques des sols étudiés.

Conclusion et application: La valorisation des déchets comme source des matières organiques est une pratique à encourager en horticulture urbaine à Lubumbashi étant donné le coût élevé des engrais minéraux et le faible niveau de revenu des agriculteurs.

Mots clés : Compost de fumiers de poules, engrais minéraux, sol acide, agriculture urbaine et périurbaine et Lubumbashi. 


\section{ABSTRACT}

\section{Improvement quality of soils acids of Lubumbashi (Katanga, Congo) by applying different levels of chicken manure compost}

Objectives: An experiment was conducted on an acidic soil to assess the influence of compost and mineral fertilizers on the chemical properties of this soil.

Methodology and results: The test was installed to a completely randomized with six replicates of six treatment: T0 (unfertilized control), T1 (175 kg NPK+ $87 \mathrm{~kg}$ urea per hectare) T2 (double of T1, $350 \mathrm{~kg}$

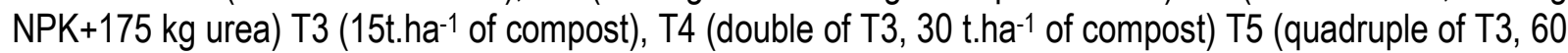
t.ha-1 of compost). Before installation of the test, composting chicken manure was carried out for 36 days. At the end of the experiment, chemical analyzes were performed on samples of soil and compost from manure of hens. The contents of exchangeable cations appear to be higher compared to the reference values found by other authors in the study area. The high values found for these parameters in the site of the present study could result from the contribution of fertilizers. For the witness, there was a low nutrient availability. In addition, the mean levels of $\mathrm{K}, \mathrm{P}, \mathrm{C}$ and pH are high in pots amended with plenty of compost. Significant differences were observed between the different doses of compost and mineral NPK fertilizer on soil characteristics studied.

Conclusion and application: Recovery of waste as a source of organic matter is a practice to be encouraged in Lubumbashi urban horticulture, in given the high cost of mineral fertilizers and the low level of income of farmers.

Keywords: Compost, mineral fertilizers, acid soil, urban and peri-urban agriculture, Lubumbashi.

\section{INTRODUCTION}

La fertilité ou richesse d'un agroécosystème se mesure par sa capacité à produire de manière performante différents produits utiles à l'homme. Le maintien de cette performance est vital tandis que sa baisse se traduit par une perte progressive des rendements [Kasongo et al., 2013]. Comme beaucoup de sols ferralitiques [Chaussod et al., 1992], les sols de Lubumbashi sont pauvres et le rôle de la matière organique n'est plus à démontrer pour ces sols [Kasongo et al., 2013]. La mise en culture de ces sols conduit à une chute rapide de matières organiques et un effondrement de la fertilité tant chimique que biologique [Mulaji, 2011], alors que d'ici 2030, la population mondiale augmentera de 3 milliards d'individus et la production de nourriture devra doubler [Olanreju et al., 2004]. Cependant, la gestion durable de la fertilité des sols dans les zones tropicales humides demeure d'actualité, parce que la majorité de la production agricole dans les sols tropicaux reste encore basée sur le système traditionnel [N'dienor, 2006]. La recherche des alternatives porteuses de soulagement est très importante. Dans un contexte d'insécurité alimentaire, de réduction de la fertilité des sols et de la hausse des prix des engrais sur les marchés, il apparaît nécessaire d'utiliser pour l'agriculture les nutriments disponibles et à faible coût [Useni et al., 2013]. De plus, l'application exclusive des engrais minéraux n'est généralement efficace que pendant les premières années d'apports continus ; on constate en effet une baisse de rendement après quelques années à cause de la dégradation des propriétés des sols [Useni et al., 2012]. Des récentes recherches ont montré que les déchets organiques, produits en grande quantité en ville avec difficulté d'évacuation, constituent une alternative à la fertilisation minérale [Mulaji, 2011; Useni et al., 2012]. Ces déchets organiques, à l'instar des fumiers de poules, contribuent dans l'amélioration des propriétés du sol, fournissent les éléments nutritifs au sol et contribuent ainsi à l'augmentation des rendements des légumes [Mulaji, 2011] et des céréales [Kiba, 2005; Useni et al., 2012]. Toutefois, ces déchets organiques sont appliqués généralement sans compostage au préalable alors que le compostage des déchets apparaît non seulement comme une méthode prometteuse de recyclage mais aussi d'assainissement de ces déchets. Ce procédé (compostage) permet 
d'hygiéniser les biodéchets et d'amorcer une humification en une période relativement courte [N'Dayegamiye et al., 2005]. Dans la ville de Lubumbashi, l'étude de Mulang [2013] a montré un effet faible ou nul de la fertilisation minérale sur la capacité de rétention d'eau du sol et les propriétés chimiques en fin d'essai tandis que l'apport en matière organique (fumier de poule) est associé à une amélioration de la structure du sol et à un stockage de nutriments dans le sol. Cette étude a

\section{MILIEU, MATERIEL ET METHODES}

Milieu : L'expérimentation a été conduite au champ expérimental de la faculté des Sciences agronomiques de I'Université de Lubumbashi (UNILU), de coordonnées géographiques: $027^{\circ} 48^{\prime} 61^{\prime \prime} 1$ de longitude Est, $11^{\circ} 61^{\prime} 553^{\prime \prime}$ de la latitude Sud, et $1257 \mathrm{~m}$ d'altitude. La ville de Lubumbashi (figure 1) est caractérisée par un climat du type Cw6 de la classification de Koppën [FAO, 2005]. Elle est été réalisée sur un sol acide en vue d'évaluer l'effet de compost de fumiers de poules sur les propriétés chimiques par rapport aux engrais minéraux. Les objectifs spécifiques étant d'évaluer les effets des composts sur les propriétés du sol et de déterminer la dose optimale. Les hypothèses émises sont: (i) le fumier de poule est une source d'éléments fertilisants et améliore les propriétés chimiques du sol et (ii) il existe une dose optimale à l'application du compost de fumiers de poules.

caractérisée par la température moyenne annuelle est de $20^{\circ} \mathrm{C}$ [Mujinya et al., 2011]. Les mois d'octobre et de novembre sont les plus chauds, avec une moyenne de $32^{\circ} \mathrm{C}$ et $23^{\circ} \mathrm{C}$ de température maximale et minimale journalières; tandis que le mois de juillet est le plus froid, avec la moyenne des minima journaliers de $8^{\circ} \mathrm{C}$ [Vranken et al., 2013].
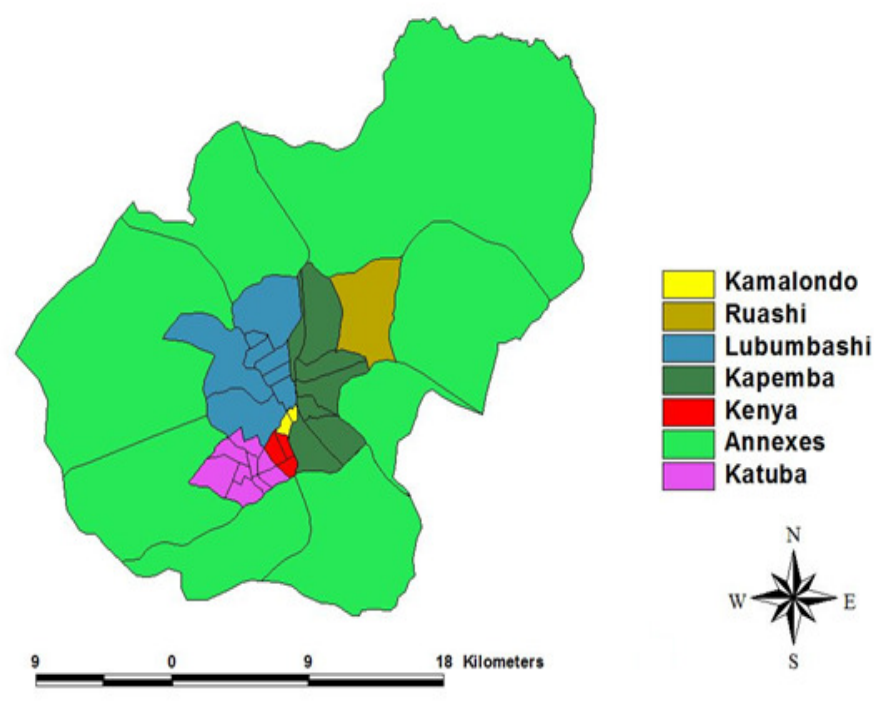

Figure 1. Subdivisions administratives de Lubumbashi

\section{MATERIEL ET METHODES}

Préparation du substrat et composition chimique : Le sol de la couche de surface $(0-30 \mathrm{~cm})$ d'un bloc du champ expérimentale de l'UNILU a été utilisé comme substrat d'étude. La couverture herbacée du bloc utilisé pour le prélèvement de ce sol, était faite de Bidens pilosa, Bidens oligoflora, Ancathospermum hispidum, Panicum maximum, Imperata cylindrica et Cynodon dactylon, qui recouvraient le terrain à des degrés variés. Ce matériel est un ferralsol de la série Katuba, selon la classification de l'INEAC [Mpundu, 2010], et dont l'analyse morphologique a révélé une texture argilo-sableuse. Ce sol était caractérisé par un $\mathrm{pH}$ de 4,$6 ; 0,001 \%$ de $\mathrm{P} ; 0,34 \% \mathrm{~N} ; 4,21 \%$ de $\mathrm{K} ; 7,21 \%$ de $\mathrm{C} ; 0,087 \%$ de $\mathrm{Ca}$ et un rapport $\mathrm{C} / \mathrm{N}$ de 21,2 . Trois $\mathrm{kg}$ 
du sol ainsi conditionné ont été mis dans chaque unité expérimentale, avant application des traitements proprement dits.

Compostage et caractéristiques des composts obtenus: Le fumier de poules obtenu à la ferme DAIPN/station Kilobelobe a été utilisé comme amendement organique après compostage. Des sachets plastic de $5 \mathrm{~kg}$ de capacité ont été utilisés comme bac de compostage; et pour éviter les pertes des éléments solubles par lixiviation, l'étanchéité de ces bacs de compostage a été observée. Après analyse au laboratoire, les résultats ont montré que la composition chimique était de $3,25 \% \mathrm{P} ; 5,6 \% \mathrm{~N}$; $7,046 \%$ de $\mathrm{K}, 19,24 \%$ Ca et un rapport $\mathrm{C} / \mathrm{N}$ de 5 . L'Urée (46\% d'azote) et le NPK 17-17-17 ont été utilisées comme fertilisants minéraux.

Conduite de l'essai, paramètres analysés et traitements de données: Cette étude a conduite en pots de plastique en polyéthylène et installé suivant un dispositif complètement randomisé avec six répétitions. Six traitements ont été préconisés, comprenant notamment 3 traitements organiques constitués des

\section{RESULTATS}

Les apports des composts ont un effet significatif sur la teneur en phosphore dans le sol. Cet effet est fonction des doses apportées et de type de fertilisants. Les résultats de l'ANOVA montrent qu'il existe des différences significatives $(p<0,00)$ entre traitements appliqués (Figure 2). II en résulte que le traitement témoin comme le traitement à la forte dose des engrais minéraux ainsi que celui à dose moyenne de composts composts de fumiers de poules $\left(15\right.$ t.ha $^{-1}, 30$ t.ha $^{-1}$ et 60 t.ha $^{-1}$ ) 2 traitements minéraux constitué d'un mélange d'urée et de NPK 17-17-17 (87 kg.ha-1 urée + 350 kg.ha-1 NPK, et 43,5 kg.ha-1 urée + 176 kg.ha-1 NPK) un témoin sans apport extérieur. Tout au long de la période de conduite de l'essai (45jours), une humidité suffisante a été garantie au sein des unités expérimentales de $5 \mathrm{~kg}$ de sol chacun, notamment, par des arrosages à la dose journalière de $500 \mathrm{ml}$ d'eau par unité, ceci pour maintenir l'humidité des unités expérimentales. Avant et après installation de l'essai, les échantillons des sols ont été analysés au laboratoire de l'office Congolais de Contrôle (OCC) en vue de déterminer les teneurs en $\mathrm{pH}$ eau, l'Azote disponible, le Phosphore disponible, le Potassium et le Calcium disponibles dans le sol suivant les méthodes décrites par Mulaji (2011). L'Anova à un facteur a été utilisé pour évaluer les effets des composts sur les propriétés chimiques et physiques du sol, avec test post hoc de TUKEY $(P=0,05)$ en cas de différences entre les traitements. Le logiciel Minitab a été utilisé à cette fin.

ont donné un faible taux du phosphore. Au regard des comparaisons établies, les traitements à base des fortes doses de composts et ceux à faible dose de composts et des engrais minéraux ont donné un taux de phosphore similaire et élevé par rapport aux traitements témoin, à base des forte dose des engrais minéraux et dose moyenne de composts de fumiers de poules.

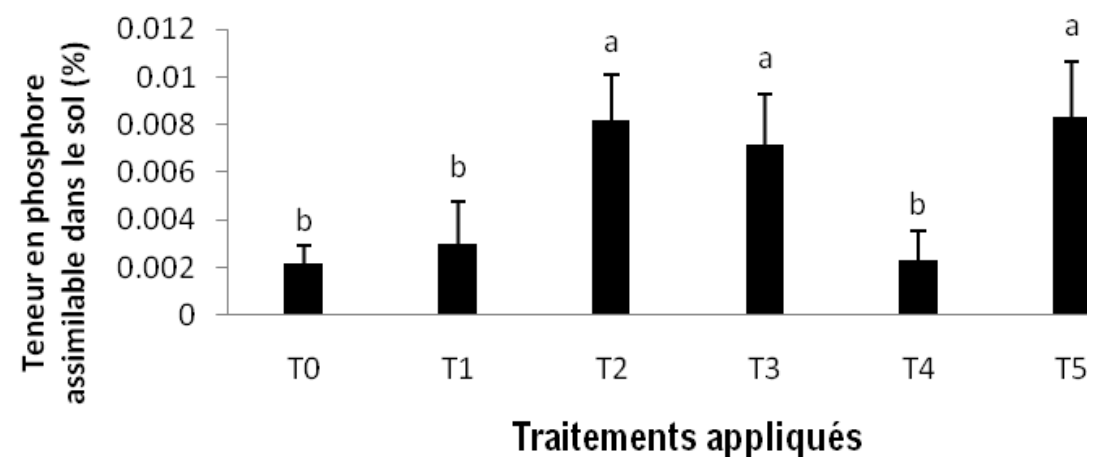

Figure 2. Teneur en phosphore disponible dans le sol en fonction des traitements. T0 : témoin ; T1 :87kg d'urée $+350 \mathrm{~kg}$ de NPK Ha-1; T2:43.5kg d'urée + 176kg de NPK Ha-1; T3:15t.ha-1 de compost des fumiers de poule ; T4 :30 tha-1 de compost des fumiers de poule; $T 5: 60$ tha $^{-1}$ de compost des fumiers de poule. 
Les résultats de l'ANOVA à un facteur contrôlé indiquent qu'il existe des différences très significatives $(p<0,000)$ en ce qui concerne le taux de matière organique dans le sol. Le taux le plus élevé a été obtenu sur le traitement à la forte dose de compost alors que les traitements à base d'engrais minéraux ont donné des taux de matière organique similaires à celui du témoin (Figure 3).

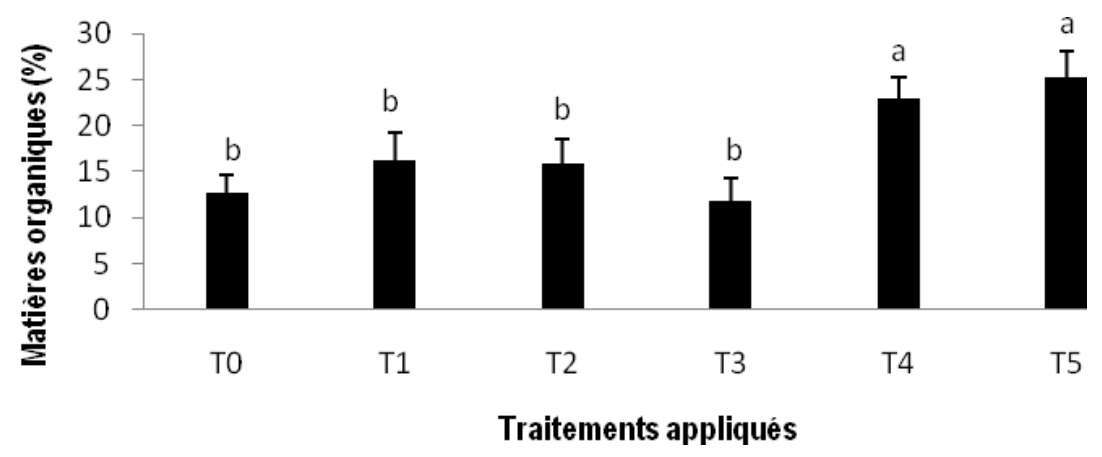

Figure 3 : Teneur en matière organique disponible dans le sol en fonction des traitements. T0 : témoin ; $\mathrm{T} 1: 87 \mathrm{~kg}$ d'urée $+350 \mathrm{~kg}$ de NPK Ha-1; T2:43.5kg d'urée + $176 \mathrm{~kg}^{-1}$ de NPK Ha-1; T3:15 t.ha-1 de compost des fumiers de poule ; T4 :30 tha-1 de compost des fumiers de poule; T5 : 60 t.ha-1 $^{-1}$ de compost des fumiers de poule.

Les résultats de l'ANOVA qui portent sur la teneur en Azote dans le sol ont montré qu'il existe de différences hautement significatives entre les différentes doses de fertilisants appliqués. Le test de Tukey qui a permis de classer les moyennes des traitements en 5 groupes (a, $b$, bc, cd et d), montre que la faible valeur a été obtenue avec le traitement témoin et la valeur la plus élevée avec le traitement à forte dose d'engrais minéraux. Par ailleurs, en comparant les traitements à forte dose de compost et à dose moyenne de compost et celui à faible dose des engrais minéraux, les résultats montrent que la faible dose d'engrais minéraux a donné une teneur faible d'Azote dans le sol (Figure 4).

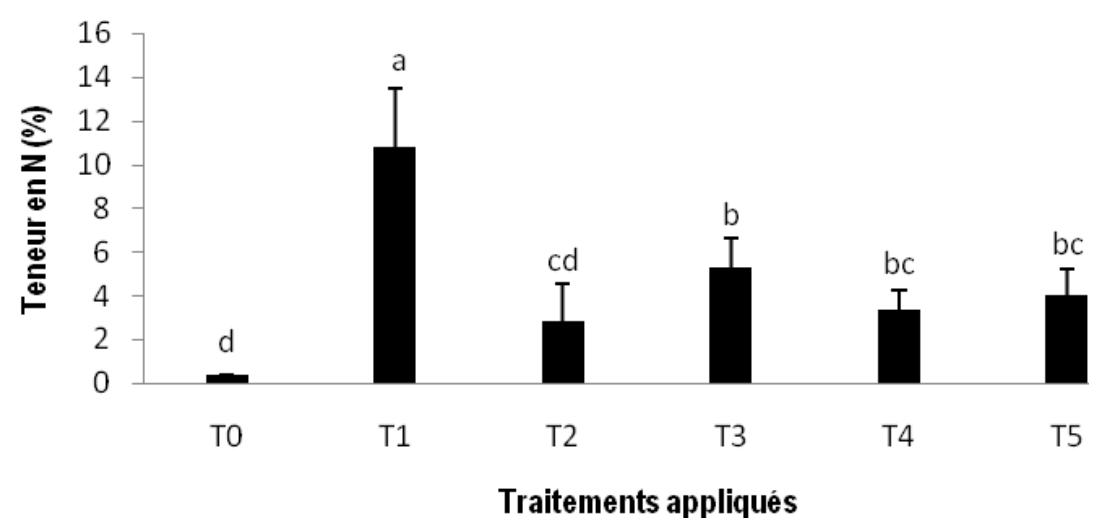

Figure 4. Teneur en Azote dans le sol en fonction des traitements. T0 : témoin ; T1 :87kg d'urée $+350 \mathrm{~kg}$ de NPK Ha1; T2 :43.5kg d'urée + $176 \mathrm{~kg}$ de NPK Ha-1; T3:15 tha- ${ }^{-1}$ de compost des fumiers de poule ; T4:30 tha ${ }^{-1}$ de compost des fumiers de poule; T5 : 60 t.ha-1 $^{-1}$ de compost des fumiers de poule.

L'effet des doses croissantes de composts et des engrais minéraux sur la teneur en $\mathrm{K}$ est présenté sur la figure 5. 


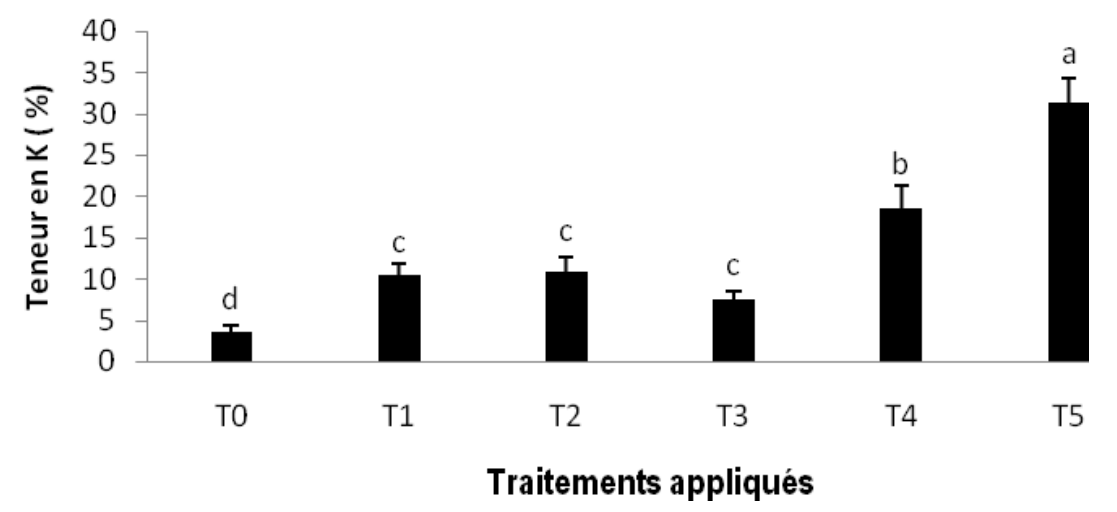

Figure 5. Teneur en Potassium dans le sol en fonction des traitements. T0: témoin; $T 1: 87 \mathrm{~kg}$ d'urée $+350 \mathrm{~kg}$ de NPK Ha-1; T2 :43.5kg d'urée + 176kg de NPK Ha-1; T3:15 tha- ${ }^{-1}$ de compost des fumiers de poule ; T4 :30 t.ha-1 de compost des fumiers de poule; $T 5: 60$ t.ha-1 $^{-1}$ de compost des fumiers de poule.

Les résultats de I'ANOVA à un facteur contrôlé indiquent qu'il existe des différences significatives $(p<$ $0,000)$ entre traitements pour ce qui est de la teneur en Potassium dans le sol. Le test de Tukey montre que la teneur en $\mathrm{K}$ la plus élevée a été obtenue avec le traitement à la forte dose de compost de fumier de poules, suivi du traitement à dose moyenne des composts de fumiers de poules; les traitements à faible dose de compost et à engrais minéraux ont donné une teneur en potassium similaires. Le traitement témoin a présenté la teneur la plus faible de cette expérimentation. Ces résultats indiquent ainsi que les apports des composts des fumiers de poules favorisent l'augmentation de la teneur en potassium dans le sol. La figure 6 montre que la variation du taux du carbone dans le sol est fonction des doses croissantes de composts de fumiers de poules et des engrais minéraux $(p<0,000)$. Le taux de carbone le plus élevé a été obtenu sur les traitements à la forte dose et à moyenne dose de compost de fumiers de poules, les traitements à base d'engrais minéraux ont donné un taux de matière organique similaires comparativement au témoin et à celui à faible dose des composts de fumiers de poules.

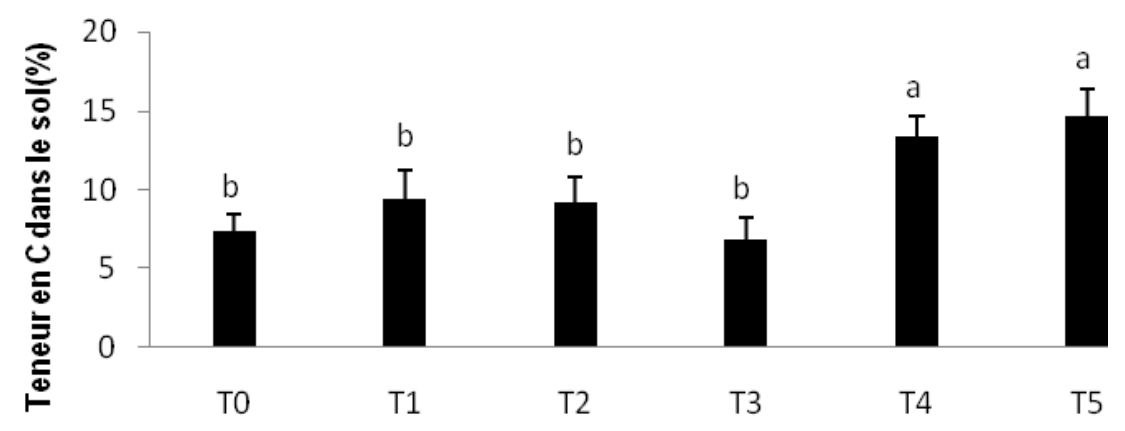

Traitements apliqués

Figure 6. Teneur en carbone dans le sol en fonction des traitements. T0 : témoin ; T1 :87kg d'urée $+350 \mathrm{~kg}$ de NPK $\mathrm{Ha}^{-1} ; \mathrm{T} 2: 43.5 \mathrm{~kg}$ d'urée $+176 \mathrm{~kg}$ de NPK Ha-1; T3:15 tha- ${ }^{-1}$ de compost des fumiers de poule; T4:30 t.ha-1 de compost des fumiers de poule; $T 5: 60$ t.ha-1 $^{-1}$ de compost des fumiers de poule.

Pour ce qui la teneur du calcium dans le sol, les résultats de l'ANOVA ont montré qu'il existe de différences significatives entre les différents type de fertilisants utilisé et les doses de compost de fumiers de poules (figure 7). Le test de Tukey montre que les valeurs extrêmes ont été obtenues avec le traitement 
témoin et le couple des traitements à dose moyenne et à forte dose de compost de fumiers de poules. Par ailleurs, les traitements à base des engrais minéraux présentent des faibles teneurs en $\mathrm{Ca}$. Ces résultats montrent que l'apport de composts de fumiers de poules augmente la teneur en Calcium dans le sol.

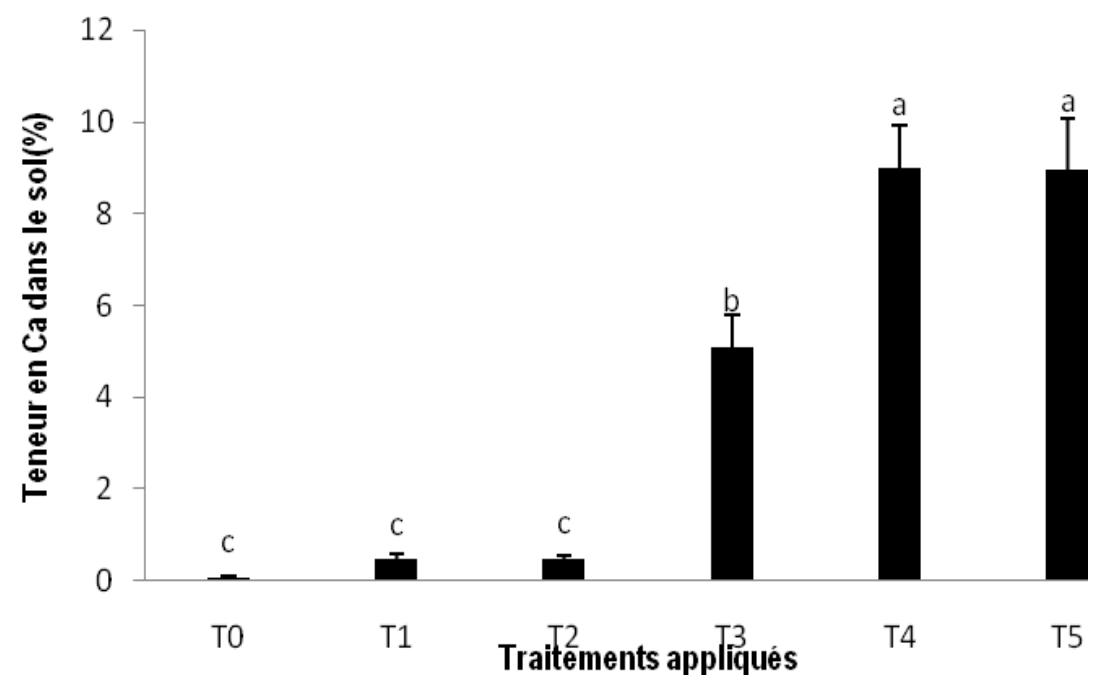

Figure 7 : Teneur en calcium dans le sol en fonction des traitements. T0 : témoin ; T1:87kg d'urée $+350 \mathrm{~kg}$ de NPK $\mathrm{Ha}^{-1} ;$ T2:43.5kg d'urée + 176kg de NPK Ha-1; T3:15 tha- ${ }^{-1}$ de compost des fumiers de poule; T4:30 t.ha-1 de compost des fumiers de poule; $\mathrm{T} 5: 60$ t.ha-1 de compost des fumiers de poule.

Quant au $\mathrm{C} / \mathrm{N}$, les résultats de l'ANOVA ont montré qu'il existe de différences significatives entre les doses de fertilisants appliqués (figure 8). Le traitement témoin présente une forte valeur du rapport carbone-azote et la faible valeur est obtenue avec le traitement à forte dose d'engrais minéraux. Par ailleurs, les traitements à base de composts de fumiers de poules présentent des résultats similaires.

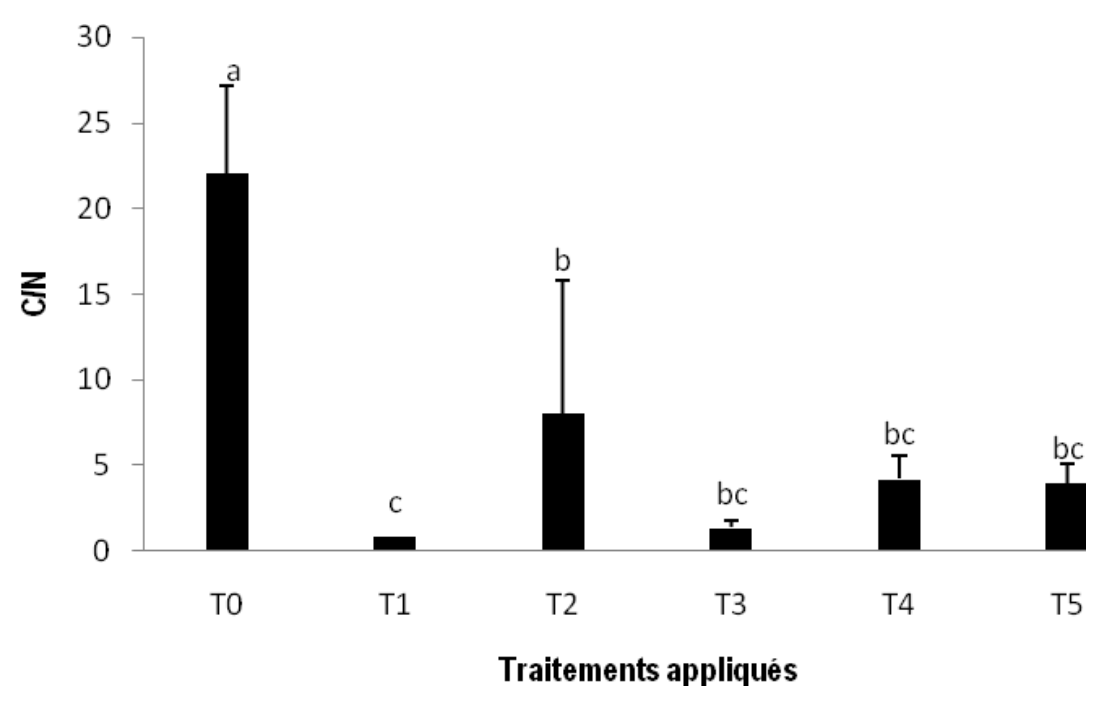

Figure 8. Rapport carbone-azote du sol en fonction des traitements. T0 : témoin ; T1 :87kg d'urée $+350 \mathrm{~kg}$ de NPK $\mathrm{Ha}^{-1} ;$ T2:43.5kg d'urée + $176 \mathrm{~kg}$ de NPK Ha-1; T3:15 tha- ${ }^{-1}$ de compost des fumiers de poule; T4:30 t.ha-1 de compost des fumiers de poule; $\mathrm{T5}: 60$ t.ha-1 $^{-1}$ de compost des fumiers de poule. 
Les résultats qui sont illustrés sur la figure 9 montrent que le $\mathrm{pH}$ a varié entre 4,7 pour le traitement témoin et 5,6 pour le traitement à base des composts de fumiers de poules. Les résultats de l'ANOVA montrent que $(p<$ 0,00 ) les traitements témoin et ceux à base des engrais minéraux présentent des valeurs de $\mathrm{pH}$ similaires et faibles par rapport aux traitements à base des composts de fumier de poules. Par ailleurs les traitements à base des fortes doses de composts ont donné une valeur de $\mathrm{pH}$ plus élevé que les traitements doses moyenne et forte dose de compost de fumier de poules.

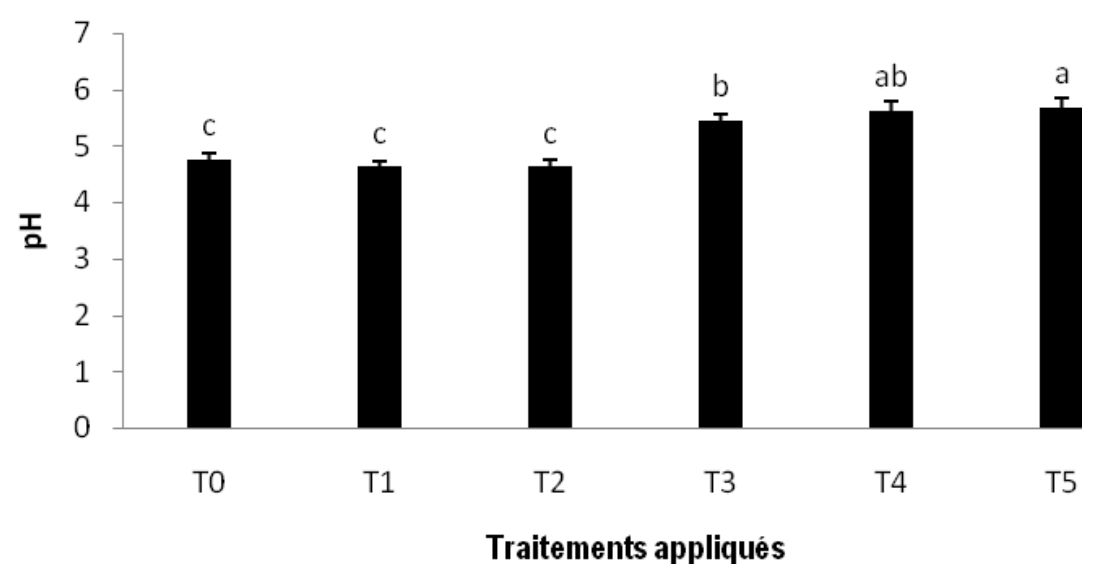

Figure 9 : Variation du pH du sol en fonction des traitements. T0 : témoin ; T1 :87kg d'urée +350kg de NPK Ha-1; T2 :43.5kg d'urée + 176kg de NPK Ha-1; T3:15t/ha de compost des fumiers de poule ; T4:30 t/ha de compost des fumiers de poule; $T 5: 60 t / h a$ de compost des fumiers de poule.

\section{DISCUSSION DES RESULTATS}

Les teneurs en cations échangeables semblent plus élevées par rapport aux valeurs de référence trouvées par d'autres auteurs dans la zone d'étude (Kasongo, 2008 ; Mukalay et al., 2008 ; Mpundu, 2010). Les valeurs élevées trouvées pour ces paramètres dans le site de la présente étude pourraient provenir de l'apport des fertilisants. Par contre, sur le témoin, l'on a noté une faible disponibilité des éléments nutritifs. Nyembo et al. (2014) indiquent que la faible efficience agronomique limite l'utilisation de l'engrais dans les ménages des agriculteurs africains; mais elle pourrait être améliorée grâce à une gestion complémentaire de la structure du sol et des matières organiques. Les résultats obtenus du présent essai indiquent que l'ajout de compost augmente la quantité de phosphore disponible pour les plantes, tel que démontré par Kiba (2005). Les résultats similaires ont été observés par Mulaji (2011) avec un compost à base des déchets organiques sur un ferralsol fortement acide. Selon, Mulaji (2011), le devenir du phosphore ou sa disponibilité dans un sol amendé en compost est plus dépendant des propriétés intrinsèques du sol (teneur en phosphore échangeable, pouvoir fixateur, concentration des ions phosphatées en solution) que de la minéralisation de la matière organique $d u$ compost. Pour ce qui est de l'azote, les composts contiennent relativement peu d'azote $(0,5$ à $6 \%$ en fonction des composants de base), leur apport en quantité peut fournir l'azote complémentaire nécessaire et enclencher sa dynamique dans le sol (N'dienor, 2006). Les fumures organiques libèrent de l'azote sous forme assimilable pour la plante et leurs actions sont lentes et progressives (Nyembo et al., 2014). Ainsi, quand le compost est apporté au sol, l'azote est, la première année, surtout sous forme organique, alors qu'il se minéralise de plus en plus les années suivantes. La valeur fertilisante de l'azote est donc en général faible mais à ne pas négliger en cas d'apport important. Par ailleurs, le compost semble accroitre la disponibilité de l'azote, tel que démontré dans une étude conduite par Useni et al. (2013) dans la ville de Lubumbashi. Les taux de réduction du carbone organique dans les pots au NPK seraient dus à la stimulation de l'activité métabolique suite à l'apport des éléments N, P, K des engrais (Kiba, 2005 ; Kaho et al., 2011). Pour le témoin et les amendements aux NPK, on enregistre des pertes beaucoup importantes. Ceci s'expliquerait du fait que ces traitements n'ont pas reçu d'apports en matière organique et en plus ces pertes peuvent être amplifiées par une minéralisation intense 
compte tenue des conditions climatiques (fortes précipitations et températures) (Meng et al., 2005; Kaho et al., 2011). Ceci peut entrainer, à long terme en l'absence de tout apport, une perte significative des stocks organiques avec comme conséquence la détérioration de la qualité de sol (Kaho et al., 2011) . Des fortes quantités de ces nutriments apportés par la matière organique sont associées avec les particules inorganiques et organiques solides $\mathrm{du}$ sol. Ces différents cations étant libérés au rythme de la minéralisation sont fonction des apports totaux. Beaucoup d'études ont montré un accroissement considérable des concentrations des cations basiques principaux $(\mathrm{Ca}, \mathrm{K})$ dans le sol après un apport répété en compost (N'Dayegamiye et al., 2005 ; Kotchi et al., 2010 ; Mulaji, 2011). Pour le pH, l'apport des matières organiques par leurs contenus en bioéléments minéraux ( $\mathrm{Ca}$ et $\mathrm{Mg}$ ), leur grande capacité d'échange cationique et de leur effet tampon rehausse le $\mathrm{pH}$ du sol (Shutcha et al., 2010). Cette augmentation serait due non seulement à l'effet tampon de la matière organique, à la teneur en cations basiques (essentiellement le $\mathrm{Ca}$ et $\mathrm{Mg}$ ), à la présence des microorganismes filamenteux qui prennent naissance dans les apports organiques et à la complexation de l'aluminium (Kasongo, 2008 ; Mukalay et al., 2008).

\section{CONCLUSION}

Les niveaux moyens du potassium et du phosphore sont élevés dans les sols amendés avec de grandes quantités de composts. Le $\mathrm{pH}$ et le carbone augmentent également avec la dose appliquée de la matière organique des composts. Les différences significatives ont été observées entre les différentes doses des composts utilisés et des engrais minéraux NPK sur les caractéristiques des sols étudiés quant à leurs effets. La différence entre la dose T4 $\left(30\right.$ t.ha $\left.^{-1}\right)$ et T5 (60 t.ha-1) n'est significative pour la plupart des paramètres étudiés. Les résultats montrent une certaine efficacité des engrais minéraux utilisés pour certains paramètres, mais tendent à dégrader d'autres. Bien que, les apports en matières organiques des composts semblent améliorer les propriétés chimiques des sols, l'étude montre que le niveau de fertilité obtenu

\section{REFERENCES BIBLIOGRAPHIQUES}

Chaussod R., Zuvia M., Breuil M.C., Hetier J.M., 1992. Biomasse microbienne et "statut organique " des sols tropicaux : exemple d'un sol vénézuélien de Llonos sous différents
Les valeurs trouvées pour l'azote sont caractéristiques des sols à faible teneur en azote, due probablement à la faible vitesse de minéralisation de la matière organique (Kaho et al., 2011). Selon, Mulaji (2011), dans les sols à $\mathrm{pH}<5,5$; les bactéries nitrifiantes et fixatrices d'azote sont détruites par l'acidité du sol, et la nitrification de la matière organique est significativement limitée, conduisant ainsi la déficience en azote. La baisse du $\mathrm{pH}$ dans les parcelles ayant reçu des engrais chimiques corrobore les résultats de travaux antérieurs qui ont révélé l'effet acidifiant des engrais chimiques sur les Altisols et Ultisols au Ghana et au Nigeria (Kaho et al., 2011). Antérieurement, la différence dans la gamme de $\mathrm{pH}$ a été identifiée comme l'un des facteurs principaux à la base de cette hétérogénéité des plants de maïs et de leurs rendements à Lubumbashi (Mukalay et al., 2008) . En plus, Shutcha et al. (2010) montrent que le pH est le principal facteur de mobilité des métaux dans le Sol. En effet, l'acidité des sols a une très grande influence sur la concentration des éléments nutritifs et toxiques de la solution du sol. L'augmentation du $\mathrm{pH}$ suite à l'apport des composts de fumiers de poules est une bonne option pour la restauration de la fertilité des sols dans la ville de Lubumbashi (Useni et al., 2014), étant donné que la carence en nutriments est liée à la faible mobilité des éléments nutritifs et la forte mobilité de l'Al.

reste fragile dans les cas des sols acides tropicaux sableux en plus des conditions climatiques qui prévalent dans le milieu, caractérisées par des fortes précipitations et des températures élevées. De ce point de vue, il est important de mettre en place un système de gestion qui assure la conservation des sols et leur utilisation rationnelle dans le but d'une agriculture soutenable. La valorisation des déchets comme source des matières organiques doit être encouragée dans le système global de gestion des déchets dans la ville de Lubumbashi. Enfin, le suivi des paramètres chimiques ne suffira pas pour prédire de l'état de santé d'un sol dans un écosystème donné, il conviendrait aux autres chercheurs d'approfondir cette étude pour suivre les paramètres physique et biologiques.

systèmes de culture. Cah. Orstom., sér. Pédol., 27(1) : 59-67

FAO, 2005. New_LocClim: Local Climate Estimator. FAO Environment and Natural Resources Working Paper, $\mathrm{N}^{\circ} 20$. 
Kaho F., Yemefack M., Feujio-Tegwefouet P., Tchanthaouang J.C., 2011. Effet combiné de feuilles de Tithonia diversifolia et des engrais inorganiques sur le rendement du maïs et les propriétés d'un sol ferralitique au centre du Cameroun. Tropicultura, 29(1):39-45

Kasongo L.M.E., 2008. Système d'évaluation des terres à multiples échelles pour la détermination de l'impact de la gestion agricole sur la sécurité alimentaire au Katanga, RD Congo. Thèse de doctorat, Université de Gand. 309 p.

Kasongo L.M.E., Mwamba M.T., Tshipoya M.P., Mukalay M.J., Useni S.Y., Mazinga K.M., Nyembo K.L., 2013. Réponse de la culture de soja (Glycine max L. (Merril) à l'apport des biomasses vertes de Tithonia diversifolia (Hemsley) A. Gray comme fumure organique sur un Ferralsol à Lubumbashi, R.D. Congo. Journal of Applied Biosciences 63: 47274735

Kiba D.I., 2005. Valorisation agronomique des excréta humains : utilisation de fèces humaines et des urines pour la production de l'aubergine (Solanum melongena) et du maïs (Zea mays) dans la zone centre du Burkina Faso. Mémoire de Fin d'études, Université Polytechnique de Bobo Dioulasso, Institut de Développement Rural, 79p.

Kotchi V., Yao K.A., Sitapha D., 2010. Réponse de cinq variétés de riz à l'apport de phosphate naturel de Tilmesi (Mali) sur les sols acides de la région forestière de Man (Côte d'lvoire). Journal of applied Bioscience 31 : 1895-1905

Mbonigaba M.J.J., 2007. Etude de l'impact des composts à base de biomasse végétale sur la dynamique des indicateurs physico-chimiques, chimiques et microbiologiques de la fertilité des sols: application sur trios sols acides tropicaux du Rwanda. Thèse de doctorat, FUSAGX, Gembloux, 243p

Meng L., Ding W., Cai Z., 2005. Long-term application of organic manure and nitrogen fertilizer on NO2 emission, soil quality and crop production in a sandy loam soil. Soil Biol. Biochem. 37 : $2037-2045$

Mpundu M.M., 2010. Contamination des sols en Éléments Traces Métalliques à Lubumbashi (Katanga/RD Congo). Évaluation des risques de contamination de la chaîne alimentaire et choix de solutions de rémédiation. Thèse de doctorat, Faculté des sciences agronomiques, Université de Lubumbashi, 432p

Mujinya B.B., Mees F., Boeckx P., Bode S., Baert G., Erens H., Delefortrie S., Verdoodt A., Ngongo M.L., Van Ranst E., 2011. The origin of carbonate in the termite mounds of the Lubumbashi area, DR Congo. Geoderma, 165: 95-105

Mukalay M.J., Shutcha N.M., Tshomba K.J., Mulowayi K.A., Kamb C.F., Ngongo L.M., 2008. Causes d'une forte hétérogénéité des plants dans un champ de maïs dans les conditions pédoclimatique de Lubumbashi. Presses Universitaires de Lubumbashi, Annales Faculté des Sciences Agronomiques, 1(2) : 411

Mulaji K.C., 2011. Utilisation des composts de biodéchets ménagers pour l'amélioration de la fertilité des sols acides de la province de Kinshasa (République Démocratique du Congo). Thèse de doctorat, université de Liège- Gembloux Agro-Biotech, 220p

Mulang T.S., 2013. Influence de la fertilisation minérale, organique et organominérale sur les propriétés physicochimiques du sol et le comportement du maïs. Mémoire de fin d'études, Faculté des Sciences Agronomiques, Université de Lubumbashi, 50p, 2013

N'Dayegamiye A., A. Drapeau A., M.R. Laverdière M.R., 2005. Effets des apports de composts de résidus ménagers sur les rendements des cultures et certaines propriétés du sol. Agrosolutions 16(2) :vol $16 \mathrm{n}^{\circ} 2 \mathrm{pp}$ 57-71

N'Dienor M., 2006. Fertilité et gestion de la fertilisation dans les systèmes maraîchers périurbains des pays en développement : intérêts et limites de la valorisation agricole des déchets urbains dans ces systèmes, cas de l'agglomération d'Antananarivo (Madagascar). Thèse de doctorat, Université d'Antanarivo, Ecole Supérieure des Sciences Agronomiques, 242p

Nyembo K.L., Useni S.Y., Chinawej M.M.D., Kyabuntu I.D., Kaboza Y., Mpundu M.M., Baboy L.L., 2014. Amélioration des propriétés physiques et chimiques des sols sous l'apport combiné des biodéchets et des engrais minéraux et influence sur le comportement du maïs (Zea mays L. var Unilu). Journal of applied Bioscience 74 : 6221-6130

Olanreju B.S., P. Moustier P., , L. Mougeot L., Fall A., 2004. Développement durable de l'agriculture 
urbaine en Afrique francophone: enjeux, concepts et methods. CIRAD-CRDI, 173

Shutcha M.N., Mubemba M.M., Faucon M-P., Luhembwe M.N., Visser M., Colinet G., Meerts P., 2010. Phytostabilisation of CopperContaminated Soil in Katanga: An Experiment with Three Native Grasses and Two Amendments. International Journal of Phytoremediation, 12(6): $616-632$

Useni S.Y., Mwema L.A., Musambi L., Chinawej M.M.D., Nyembo K.L., 2014. L'apport des faibles doses d'engrais minéraux permet-ild'accroitre le rendement du maïs cultivé à forte densité ? Un exemple avec deux variétés de maïs à Lubumbashi. Journal of applied Bioscience 74 : 6131-6140

Useni S.Y., Chukiyabo K.M., Tshomba K.J., Muyambo M.E., Kapalanga K.P., Ntumba N.F., Kasangij K.P., Kyungu K.A., Baboy L.L., Nyembo K.L., Mpundu M.M., 2013. Utilisation des déchets humains recyclés pour l'augmentation de la production du maïs (Zea mays L.) sur un ferralsol du sud-est de la RD Congo. Journal of Applied Biosciences 66: 5070 - 50811

Useni S.Y., Baboy L.L., Nyembo K.L., Mpundu M.M., 2012. Effets des apports combinés de biodéchets et de fertilisants inorganiques sur le rendement de trois variétés de Zea mays $L$. cultivées dans la région de Lubumbashi. Journal of Applied Biosciences 54: 39353943

Vanlauwe B., Wendt J., Diels J., 2001. Combined application of organic matter and fertilizer. in Tian G, Ishida F and Keating J, Sustaining soil fertility in West Africa. Soil Sciences Society, a Special 58: $247-280$

Vranken I., Amisi M.Y., Munyemba K.F., Bamba I., Veroustraete F., Visser M., Bogaert J., 2013. The spatial footprint of the non-ferrous mining industry in Lubumbashi. Tropicultura 1: 20-27 